\title{
INFLUENCIA DE LA FUNCIÓN VISUAL EN LA CALIDAD DE VIDA DE LOS PACIENTES CON GLAUCOMA
}

\section{INFLUENCE OF VISUAL FUNCTION ON QUALITY OF LIFE IN PATIENTS WITH GLAUCOMA}

\author{
CARRASCO-FONT C ${ }^{1}$, LORENZO-MARTÍNEZ $\mathrm{S}^{1}$, GILI-MANZANARO P ${ }^{1}$, ARIAS-PUENTE A ${ }^{1}$, \\ ANDRÉS-ALBA Y ${ }^{2}$, MATILLA-RODRÍGUEZ A ${ }^{2}$, ORTIGUEIRA JA ${ }^{1}$
}

\section{RESUMEN}

Objetivo: Determinar la relación entre las medidas objetivas de la función visual y la percepción de los pacientes de su calidad de vida relacionada con la salud.

Métodos: Estudio transversal de evaluación de la calidad de vida en pacientes con glaucoma o hipertensión ocular y con un nivel intelectual suficiente para comprender el cuestionario. Período de estudio: abril-mayo de 2006. Ambito: hospital de tercer nivel de la Comunidad de Madrid. Instrumento: Índice de Función Visual VF-14.

Se registró agudeza visual, perimetría computerizada, y exploración oftalmológica completa.

Resultados: Se recogieron 120 cuestionarios, en los que el 89,1\% lo cumplimentó el propio paciente. Para el análisis de fiabilidad y validez de los datos se uso el índice de fiabilidad alpha de Cronbach $(0,88)$. Cuarenta y nueve pacientes fueron diagnosticados de hipertensión ocular (HTO) y 71 de glaucoma (52,1\% hombres y 47,9\% mujeres). La valoración media de su calidad de vida fue de 73,9 (DE: 24,13 R: 0-100); HTO: 81,4 (DE: 16,3) у glaucoma 68,69 (DE: 22) (p=0,001). Se han encontrado diferencias del estado de salud en relación con la agudeza visual $(\mathrm{r}=0,51)$, defecto campimétrico

\section{ABSTRACT}

Objective: To determine the relationship between visual function objective measures and the perceived quality of life in patients with glaucoma and ocular hypertension.

Method: This was a cross-sectional study of quality of life. Patients required knowledge to understand the questionnaire. Period of study: April-May 2006. Setting: third level hospital in Madrid. Instrument: Visual Function Index (VF-14). Visual acuity, computerized perimetry and ophthalmological examination were all performed.

Results: 120 questionnaires were distributed, $89.1 \%$ were returned completed. Cronbach's alpha was used for data validity and reliability $(0.88) .49$ patients had ocular hypertension (HTO) and 71 had glaucoma (52.1\% men and $47.9 \%$ women). The mean health status was 73.9 (SD 24.13; range: 0-100); HTO: 81.4 (SD: 16.3) and glaucoma: 68.69 (SD:22) $(\mathrm{p}=0.001)$. These results were significantly correlated with the quality of life and visual acuity $(\mathrm{r}=0.51)$, mean deviation (MD) $r=0.35$, education level $(p=0.024)$, and sex $(\mathrm{p}=0.031)$. No significant differences were found in the older groups or for evolution time.

Conclusions: Quality of life measures can be useful in the management of patients with glaucoma, as

\footnotetext{
Recibido: 28/2/07. Aceptado: 3/3/08.

Hospital Fundación Alcorcón. Madrid. España.

1 Doctor en Medicina.

2 Licenciado en Medicina.

Correspondencia:

Carmen Carrasco Font

Hospital Fundación Alcorcón

Avda. Budapest, 1

28922 Alcorcón (Madrid)

España

E-mail: ccarrasco@fhalcorcon.es
} 
$(\mathrm{r}=0,35)$, nivel de estudios $(\mathrm{p}=0,024)$, y sexo $(\mathrm{p}=0,031)$. No se observó peor estado de salud en los grupos de mayor edad ni con el tiempo de evolución de la enfermedad.

Conclusiones: Las medidas de calidad de vida pueden resultar útiles en la monitorización de los pacientes con glaucoma. Observamos moderada correlación entre agudeza visual, alteración campimétrica y calidad de vida de los pacientes con glaucoma. El VF-14 permite diferenciar entre pacientes con glaucoma e hipertensión ocular.

Palabras clave: Glaucoma, función visual, calidad de vida, VF-14 test, campo visual. a moderate relationship was found between quality of life, visual acuity and visual field loss in such patients. The VF-14 seems to discriminate between glaucoma and ocular hypertensive patients (Arch Soc Esp Oftalmol 2008; 83: 249-256).

Key words: Glaucoma, visual function, quality of life, VF-14 test, visual field.

\section{INTRODUCCIÓN}

El glaucoma no solo afecta a la función visual e incrementa el gasto por tratamiento, también influye en la salud general del paciente y en su calidad de vida. Desde el momento en que se diagnostica la enfermedad, por la ansiedad y el temor a la ceguera, y posteriormente por la progresión de la enfermedad, que conlleva la reducción paulatina de las actividades diarias y pérdida de confianza en sí mismos (1).

Tradicionalmente los indicadores clínicos que se utilizan para monitorizar y valorar la evolución de los pacientes con glaucoma son: presión intraocular, agudeza visual, y perimetría computerizada (2). De todos ellos, la agudeza visual y la perimetría se pueden considerar como los indicadores principales de la actividad diaria del paciente, aunque varios autores $(3,4)$ coinciden en que estas medidas pueden resultar inadecuadas como indicador del grado de afectación visual, porque no reflejan necesariamente el impacto de la enfermedad en el estado funcional total, al no tener en cuenta las actividades que el paciente puede ser capaz de realizar con su función visual residual.

Son pocos los estudios publicados en nuestro medio sobre función visual y calidad de vida, y menos aun los de glaucoma, por ello nos planteamos los siguientes objetivos:

- Determinar el efecto del glaucoma en la función visual y en la actividad diaria de los pacientes en nuestro ámbito.

- Establecer si existe relación entre las medidas objetivas de la función visual y las repuestas registradas en el cuestionario en pacientes con glaucoma e hipertensión ocular (HTO).
- Identificar si existe relación de estas medidas con el estado de calidad de vida global referente al estado de salud en el día de hoy, medido mediante una escala visual analógica (EVA) (escala de 0 a 100).

- Identificar los factores que actúan como determinantes de la calidad de vida de estos pacientes.

\section{SUJETOS, MATERIAL Y MÉTODOS}

\section{Ámbito}

Hospital de tercer nivel del Servicio Madrileño de Salud, con una población de referencia de 255.000 habitantes.

\section{Método}

Estudio observacional descriptivo transversal, a los pacientes de la consulta de glaucoma en la Unidad de Oftalmología de nuestro hospital durante marzo y abril de 2006.

\section{Instrumento de evaluación}

Cuestionario específico de Función Visual VF-14 - que valora el impacto de la enfermedad en actividades relacionadas con la visión $(5,6)$ ya validado en español (7). Consta de 20 preguntas de fácil comprensión y respuesta [escala de calificación de 0 a 5 puntos ( 0 ninguna, 1 poca, 2 bastante, 3 mucha, 4 incapaz de hacerla, 5 no la hago por razones distintas a la vista)] e incluye 6 preguntas adicionales para 
valorar el grado de visión periférica, sensibilidad al contraste y adaptación a la oscuridad. Se añadieron 5 preguntas referentes a: tratamiento, información y satisfacción global, incluyendo una escala visual analógica (EVA), (0 a 100) sobre el estado de salud en el día de hoy; y datos demográficos de los pacientes (edad, género, estado civil, nivel de estudios y situación laboral).

Se entregó el cuestionario a pacientes con diagnóstico de glaucoma o hipertensión ocular. Todos estuvieron de acuerdo en participar en el estudio y fueron capaces de rellenar el cuestionario. Para la correcta cumplimentación de ambos cuestionarios se adjuntaron unas instrucciones sencillas, en las que se hacía hincapié en la garantía del anonimato y en la necesidad de contestar con sinceridad y a ser posible no dejar ninguna pregunta sin respuesta. Aquellos pacientes con déficits sensoriales, de idioma o analfabetismo, podían ser ayudados por sus familiares o por el personal sanitario, respetando siempre la opinión del paciente.

El glaucoma fue diagnosticado por el aspecto del disco óptico y el defecto campimétrico reproducible.

Criterios de inclusión: pacientes con glaucoma primario de ángulo abierto crónico, glaucoma crónico de ángulo cerrado, o pseudoexfoliativo y pacientes con hipertensión ocular. Todos los casos estaban en tratamiento médico tópico y llevaban más de un año en tratamiento.

Criterios de exclusión: enfermedad ocular asociada que pueda provocar defectos campimétricos y visuales profundos como miopía magna, desprendimientos de retina, degeneración macular o retinopatía diabética. Así como los pacientes intervenidos de cirugía ocular en los 3 últimos meses. También se descartaron los pacientes con agudezas visuales bilaterales inferiores a 0,2 y aquellos con problemas psicológicos que no les permitían por sí mismos colaborar en el estudio. En cuanto a la agudeza visual se consideró la del ojo mejor y el defecto medio (DM) se unificó con la fórmula de sumación binocular de Nelson-Quigg (8).

Se realizó exploración oftalmológica completa a todos los pacientes incluyendo una perimetría Humphrey SITA-Fast reproducible (Carl Zeiss Systems, Dublin CA) (Swedish Threshold Interactive Algorithms). La pauta de tratamiento hipotensor ocular también fue recogida.

Para facilitar el estudio estadístico los defectos perimétricos se clasificaron según establece O’Brien (1) en tres grupos: defecto leve (defecto unilateral con pérdida menor de la mitad del campo visual), defecto moderado (unilateral mayor de medio campo o bilateral menor de la mitad en ambos ojos) y defecto severo (perdida bilateral mayor de la mitad en ambos ojos).

Para el análisis de datos se utilizó el programa de análisis estadístico SPSS 12.0 para Windows (SPSS Inc Chicago, USA). Para analizar la correlación entre el resultado del VF-14 y las demás variables se utilizó el coeficiente de correlación de Spearman. La medida de la fiabilidad del test se valoró mediante alfa de Cronbach (considera que los datos son fiables si es mayor de 0,7). El test de Kruskal Wallis se usó en las variables no paramétricas. Para el estudio de la asociación entre variables cualitativas se utilizó el test del Chi cuadrado $\left(\chi^{2}\right)$. El valor de $\mathrm{p}$ menor o igual a 0,05 fue considerado estadísticamente significativo.

\section{RESULTADOS}

Se recogieron 120 encuestas (98\%), cinco pacientes rehusaron participar. Para el análisis de fiabilidad y validez de los datos se utilizó alpha de Cronbach, obteniéndose resultados fiables y válidos: 0,88 en el grupo global; 0,84 en HTO y 0,90 en glaucoma. La media de edad de los pacientes encuestados fue de 65,58 años (DE: 10,7). Del total de pacientes $52 \%$ eran hombres y $47,9 \%$ mujeres. 49 pacientes fueron diagnosticados de hipertensión ocular (HTO) y 71 de glaucoma.

Con relación al tiempo de evolución de la enfermedad, el 50\% llevaba menos de 5 años desde el diagnóstico y $21 \%$ más de 10 años (tabla I).

Del total de pacientes $10,8 \%$ prefirió el tratamiento por la mañana y $28,2 \%$ por la noche, mientras que para el 58,3\% resultaba indiferente.

Respecto al «tratamiento que olvida con mayor frecuencia»: «por la mañana» el 22,5\%, «noche» $12,5 \%$, y los que «no lo olvidan nunca» $25 \%$.

El «tratamiento que les resultaba más sencillo» fue: «una gota al día» el $32,5 \%$ y al $52,5 \%$ no le importa el número de gotas. Los resultados respecto a los días a la semana que los pacientes olvidaban poner el tratamiento fueron: 1 día el 19,2\%, 2 días el $11,7 \%$, y no lo olvidaban nunca el $66,4 \%$. No encontramos diferencias significativas entre los pacientes con glaucoma e hipertensión ocular (tabla II).

El 69\% de los pacientes encuestados consideró que tenía suficiente información sobre su enfermedad. 
Tabla I. Características demográficas de los pacientes con glaucoma e hipertensión ocular

\begin{tabular}{|c|c|c|c|}
\hline & $\begin{array}{c}\text { Total } \\
\mathrm{n}=120\end{array}$ & $\begin{array}{l}\text { HTO } \\
n=49\end{array}$ & $\begin{array}{c}\text { Glaucoma } \\
n=71\end{array}$ \\
\hline Edad & $65,58 \mathrm{DE} 1,7$ & 63,72 DE 9 & $66,87 \mathrm{DE} 11,5$ \\
\hline Sexo: hombre/mujer & $52,1 \% / 47,9 \%$ & $52,1 \% / 47,9 \%$ & $52,1 \% / 47,9 \%$ \\
\hline AV mejor ojo & 0,73 DE 0,2 & 0,82 DE 0,19 & 0,66 DE 0,2 \\
\hline MD binocular & 10,19 DE 10 & $2,69 \mathrm{DE} 1,98$ & $15,44 \mathrm{DE} 10$ \\
\hline Cirugía previa SÍ/NO & $24,1 \% / 75,9 \%$ & $4,4 \% / 95,6 \%$ & $37,3 \% / 62,7 \%$ \\
\hline \multicolumn{4}{|l|}{ Estado civil: } \\
\hline Soltero & $21,4 \%$ & $12,5 \%$ & $27,5 \%$ \\
\hline Casado & $45,2 \%$ & $52,1 \%$ & $40,6 \%$ \\
\hline Viudo & $21,4 \%$ & $16,7 \%$ & $24,6 \%$ \\
\hline Separado & $12,0 \%$ & $18,8 \%$ & $7,2 \%$ \\
\hline \multicolumn{4}{|l|}{ Campimetría: } \\
\hline Normal & $40,7 \%$ & $97,9 \%$ & $1,4 \%$ \\
\hline Defecto leve & $22,9 \%$ & $2,1 \%$ & $37,1 \%$ \\
\hline Defecto moderado & $19,5 \%$ & & $32,9 \%$ \\
\hline Defecto severo & $16,9 \%$ & & $28,6 \%$ \\
\hline \multicolumn{4}{|l|}{ Tiempo de evolución: } \\
\hline$<5$ años & $50,0 \%$ & $54,2 \%$ & $47,1 \%$ \\
\hline 5-10 años & $28,4 \%$ & $37,5 \%$ & $22,1 \%$ \\
\hline$>10$ años & $21,6 \%$ & $8,3 \%$ & $30,9 \%$ \\
\hline
\end{tabular}

HTO: hipertensión ocular.

La valoración media de su estado de salud fue de 73,9 (DE: 24,13; R: 0-100). HTO: 81,40 (DE: 16,2)

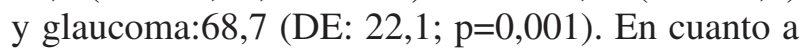
los resultados de la EVA: 65,9 (DE: 16) en glaucoma y 72,2 (DE: 17) en HTO $(\mathrm{p}=0,09)$.
Se observó una moderada correlación entre el resultado del VF-14 y la agudeza visual del ojo mejor $(\mathrm{r}=0,51)$ y con el defecto medio campimétrico $(\mathrm{r}=-$ $0,35)$. El defecto campimétrico bilateral estaba relacionado con el resultado ( $\mathrm{p}=0,0001)$ (tabla III). Se

Tabla II. Respuesta a las cuestiones sobre tratamiento tópico

\begin{tabular}{|c|c|c|c|}
\hline & Global & HTO & Glaucoma \\
\hline \multicolumn{4}{|l|}{ 1. ¿Cuándo prefiere el tratamiento? } \\
\hline Mañana & $1311,0 \%$ & $612,5 \%$ & $710,0 \%$ \\
\hline Noche & $3529,7 \%$ & $1735,4 \%$ & $1825,7 \%$ \\
\hline No importa & $7059,3 \%$ & $2552,1 \%$ & $4564,3 \%$ \\
\hline \multicolumn{4}{|c|}{ 2. ¿Cuándo se olvida con más frecuencia? } \\
\hline Mañana & $2722,7 \%$ & $1122,4 \%$ & $1622,9 \%$ \\
\hline Noche & $1512,5 \%$ & $714,3 \%$ & $811,4 \%$ \\
\hline No importa & $4739,5 \%$ & $1938,8 \%$ & $2840,0 \%$ \\
\hline No se olvidan nunca & $3025,2 \%$ & $1224,5 \%$ & $1825,7 \%$ \\
\hline \multicolumn{4}{|l|}{ 3. ¿Cuántas gotas/día prefiere? } \\
\hline 1 gota al día & $3933,3 \%$ & $1734,7 \%$ & $2231,9 \%$ \\
\hline 2 gotas al día & $1412,0 \%$ & $612,2 \%$ & $811,6 \%$ \\
\hline 3 gotas al día & $1 \quad 0,9 \%$ & & $1 \quad 1,4 \%$ \\
\hline No le importa el número de gotas & $6353,8 \%$ & $2552,1 \%$ & $3855,1 \%$ \\
\hline \multicolumn{4}{|c|}{ 4. En una semana ¿Cuántos días se olvida? } \\
\hline Una vez & $2319,3 \%$ & $1224,5 \%$ & $1115,7 \%$ \\
\hline Dos veces & $1411,8 \%$ & $510,2 \%$ & $912,9 \%$ \\
\hline Tres veces & $21,7 \%$ & $12 \%$ & $1 \quad 1,4 \%$ \\
\hline Más de tres veces & $1 \quad 0,8 \%$ & $12 \%$ & \\
\hline No se olvidan & $7966,4 \%$ & $3061,2 \%$ & $4970 \%$ \\
\hline \multicolumn{4}{|c|}{ 5. ¿Tiene suficiente información sobre su enfermedad? } \\
\hline Sí & $69 \%$ & $72,9 \%$ & $66,2 \%$ \\
\hline No & $31 \%$ & $27,1 \%$ & $33,8 \%$ \\
\hline
\end{tabular}

HTO: hipertensión ocular. 
Tabla III. Relación entre el defecto campimétrico bilateral (clasificación O'Brien) género, nivel de estudios y el nivel de estado de salud VF-14

\begin{tabular}{lcc}
\hline Resultado VF-14/Perimetría & N=118 & Rango promedio \\
\hline Normal & 47 & 71,39 \\
Leve & 28 & 61,89 \\
Moderada & 23 & 56,13 \\
Severa & 20 & 32,08 \\
\hline \hline
\end{tabular}

Kruskal-Wallis $\mathrm{p}=<0,0001$

\begin{tabular}{lcc}
\hline Resultado VF-14/ sexo & $\mathrm{N}=120$ & Rango promedio \\
\hline Genero & & \\
Hombre & 63 & 66,52 \\
Mujer & 57 & 52,90 \\
\hline \hline
\end{tabular}

Kruskal-Wallis $\mathrm{p}=0,24$

Resultado VF-14/nivel de estudios N=117 Rango promedio

\begin{tabular}{lll}
\hline Sin estudios & 25 & 42,28 \\
Estudios primarios & 53 & 61,47 \\
Bachillerato & 25 & 61,90 \\
Universitario & 14 & 44,32 \\
\hline \hline
\end{tabular}

Kruskal-Wallis $\mathrm{p}=0,31$

Tabla IV. Análisis de las variables de VF-14

\begin{tabular}{|c|c|c|c|c|c|c|}
\hline & $\mathrm{N}$ & Total & $\mathrm{N}$ & HTO & $\mathrm{N}$ & GLAU \\
\hline Letra pequeña & 101 & 59,16 & 46 & 78,48 & 55 & 48,59 \\
\hline Periódico & 106 & 66,25 & 48 & 80,01 & 58 & 56,33 \\
\hline Letra grande & 116 & 83,95 & 48 & 87,24 & 68 & 81,69 \\
\hline Reconocer personas & 119 & 87,91 & 48 & 91,32 & 71 & 85,56 \\
\hline Ver escalones & 117 & 82,91 & 49 & 91,32 & 68 & 77,11 \\
\hline Leer letreros & 116 & 82,5 & 49 & 90,30 & 67 & 77,11 \\
\hline Trabajos manuales & 107 & 70,62 & 44 & 77,04 & 63 & 66,19 \\
\hline Hacer crucigramas & 103 & 66,25 & 47 & 86,61 & 56 & 56,33 \\
\hline Jugar cartas & 104 & 77,5 & 44 & 83,16 & 60 & 73,59 \\
\hline Participar actividades & 104 & 79,16 & 44 & 84,69 & 60 & 74,64 \\
\hline Cocinar & 95 & 72,08 & 38 & 73,46 & 57 & 71,12 \\
\hline Ver TV & 117 & 84,16 & 48 & $\overline{89,28}$ & 69 & 80,63 \\
\hline Conducir día & 64 & 49,79 & 29 & 56,12 & 35 & 45,42 \\
\hline Conducir noche & 61 & $\underline{36,04}$ & 28 & 43,36 & 33 & $\overline{30,98}$ \\
\hline No chocar & 114 & $\overline{86,87}$ & 47 & $\overline{91,83}$ & 67 & $\overline{83,45}$ \\
\hline Subir bajar escalones & 118 & 82,5 & 48 & 90,30 & 70 & 77,11 \\
\hline Observar & 115 & 76,64 & 48 & 86,73 & 67 & 68,66 \\
\hline Adaptarse & 117 & 73,95 & 47 & 80,61 & 70 & 69,36 \\
\hline Ver noche & 116 & 71,87 & 49 & 80,10 & 67 & 66,19 \\
\hline Cruzar & 119 & 88,54 & 48 & 95,40 & 71 & 83,80 \\
\hline N válido (según lista) & 40 & & 19 & & 21 & \\
\hline
\end{tabular}

encontró correlación entre estado de salud y género $(\mathrm{p}=0,031)$, y el nivel de educación $(\mathrm{p}=0,024)$. No se encontraron diferencias significativas en relación a la edad ( $\mathrm{p}=0,073)$, ni al tiempo de evolución de la enfermedad $(\mathrm{p}=0,06)$.

Las variables del VF-14 (tabla IV) con medias más bajas fueron: «dificultad para leer letras pequeñas», «dificultad para leer periódico», «conducir de noche» $\mathrm{y}$ «dificultad para hacer crucigramas», que se encuentran en la categoría de visión central y cercana (excluyendo «conducir de noche» que se relaciona con luz y oscuridad).

Los resultados de todas las puntuaciones fueron inferiores en los pacientes con diagnóstico de glaucoma (fig. 1).

\section{DISCUSIÓN}

La medición de la calidad de vida relacionada con la salud (CVRS) ha alcanzado un cierto desarrollo en España (9), cada vez es más relevante

\footnotetext{
Las mejor valoradas: - Reconocer personas

- No chocar

- Cruzar la calle

Las peor valoradas: - Leer letra pequeña

- Leer el periódico

- Crucigramas

No representativas: - Conducir.
} 


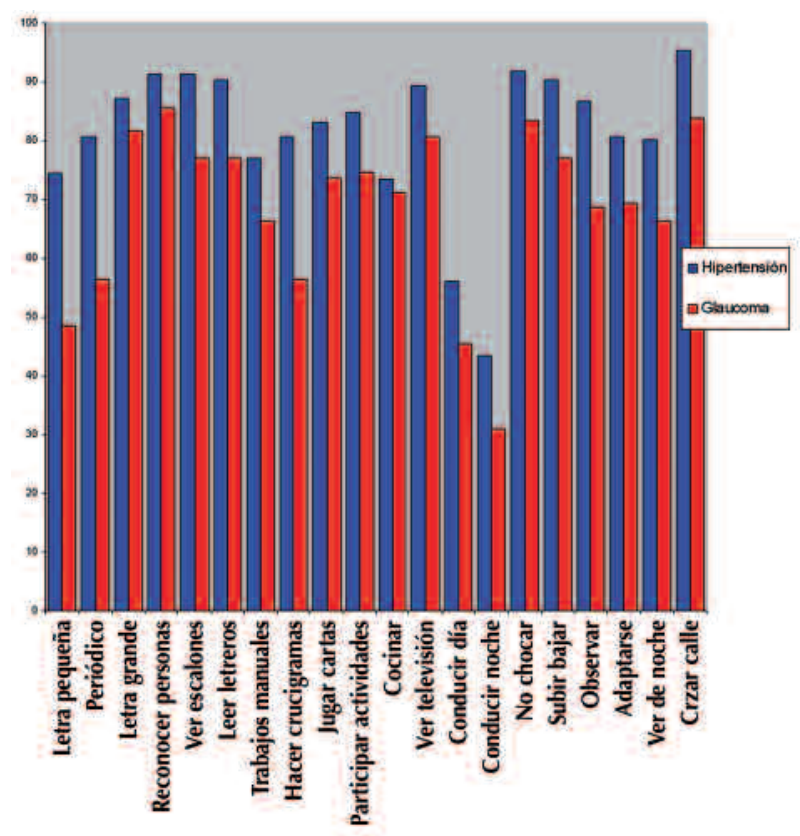

Fig. 1: Resultados del VF-14 en pacientes con glaucoma e HTO.

como una manera de estudiar la salud de la población (10) y de analizar la eficacia y la efectividad de las intervenciones sanitarias (11). Para obtener una buena medición de la CVRS se requieren cuestionarios sólidos, que puedan utilizarse de manera sencilla y rápida y que permitan obtener a la vez resultados fiables y válidos. Los cuestionarios son herramientas multidimensionales que recogen numerosos aspectos de la vida de los pacientes que no evalúan otros instrumentos diagnósticos y aportan información valiosa sobre el impacto de la enfermedad en la vida de los pacientes. Hoy día disponemos de numerosos cuestionarios, algunos de ellos genéricos, diseñados para su aplicación a la población general (12), y otros específicos, validados para grupos concretos de pacientes $(13,14)$. El valor asignado en escalas visuales analógicas puede ser utilizado como indicador cuantitativo del estado general de salud de individuo o de un grupo, de forma que permiten la comparación intra o inter individual, y constituye un método sencillo para obtener una valoración general de la propia CVRS.

El objetivo de este trabajo era evaluar la calidad de vida de pacientes con glaucoma e HTO y tener una información de cómo perciben el estado de su enfermedad en la vida diaria. Hay diversos factores que podríamos esperar que afecten a la calidad de vida como el nivel de educación, la edad $(15,16)$, el género, las condiciones socioeconómicas, el tipo de la enfermedad $(15,17)$, el tiempo de evolución $(18,19)$ o la complejidad del tratamiento (3). Por lo que los resultados podrían variar en función de la población estudiada, dependiendo de las características socioeconómicas.

Una limitación en nuestro estudio es la estimación de la correlación de la medición de la calidad de vida con los resultados clínicos, al no haber encontrado ningún cuestionario especifico para glaucoma como el SIG (The symptom impact glaucoma) (20) o el GQL-15 (The glaucoma quality of life questionnaire) (21) validados en nuestro idioma. Por ello optamos por utilizar un test genérico de función visual como el VF-14 que tiene la ventaja de ser fácil de responder, breve y reproducible $(22,23)$, aunque al no ser específico para glaucoma podría adolecer de menor correlación con los defectos campimétricos típicos en estos pacientes, pero si parece presentar adecuada discriminación en pacientes con glaucoma $(3,4,24)$ facilitándonos información sobre el impacto de la enfermedad en el manejo diario de un paciente que no es reflejado en la medida de la agudeza visual.

Otra limitación observada era que al entregar los cuestionarios a los pacientes, en ocasiones, eran los familiares los que participaban de forma activa ayudando en la elección de respuestas. Otro de los problemas encontrados es que el glaucoma es una enfermedad heterogénea con un grado de afectación y progresión muy variable de unos enfermos a otros, por lo que su estandarización resulta compleja y se requiere un número alto de casos, por lo que consideramos que, a pesar de haber obtenido una adecuada alfa de Cronbach, será necesario seguir recogiendo casos para poder realizar un estudio con una potencia estadística mayor.

Al tratarse de una enfermedad bilateral y asimétrica, en la clínica habitual seguimos monitorizando la progresión campimétrica de forma monocular, y en realidad la campimetría bilateral nos facilita mejor información sobre la situación del paciente en su vida habitual. En el análisis del defecto campimétrico hemos utilizado perimetría clásica y la clasificación de O'Brien (1) que no tiene en cuenta el campo visual bilateral con el solapamiento y corrección en ocasiones de los defectos campimétricos como ocurre con la perimetria de Esterman utilizada en trabajos similares (24).

Más de la mitad de los pacientes con glaucoma no refería ningún problema visual, los problemas más fre- 
cuentes fueron: «dificultad para leer letras pequeñas, para leer periódico, conducir de noche, y para hacer crucigramas», las cuales se encuentran en la categoría de visión central y cercana (excluyendo conducir de noche), aunque es preciso valorar que estas dificultades podrían estar mas relacionadas con la edad de los pacientes que al daño glaucomatoso en sí.

La utilización apropiada de instrumentos para valorar la calidad de vida nos puede facilitar un mayor conocimiento de la repercusión funcional de las secuelas del glaucoma en estos pacientes y de esta forma monitorizar y tratar de la forma mas adecuada al paciente de forma global y por otro lado el paciente al ser más consciente de su problema, puede participar de forma mas activa en el manejo de su enfermedad.

Los clínicos sólo nos sentiremos cómodos manejando las puntuaciones de las medidas de calidad de vida cuando éstas nos resulten tan familiares como la medición de la tensión arterial o la glucemia (14). Interesa valorar como se siente el paciente, en lugar de cómo los médicos creen que debería sentirse en función de las medidas clínicas, sobre todo en el caso de patologías crónicas.

La demostración de que los cambios en la puntuación de las medidas de la CVRS detectan cambios clínicamente importantes contribuirá a la evaluación integral y válida del estado de salud de un individuo.

\section{BIBLIOGRAFÍA}

1. Nelson P, Aspinall P, Papasouliotis $O$, Worton B, O'Brien C. Quality of life in glaucoma and its relationship with visual function. J Glaucoma 2003; 12: 139-150.

2. Odberg T, Jakobsen JE, Hultgren SJ, Halseide R. The impact of glaucoma on the quality of life of patients in Norway. I. Results from a self-administered questionnaire. Acta Ophthalmol Scand 2001; 79: 116-120.

3. Sherwood MB, Garcia-Siekavizza A, Meltzer MI, Hebert A, Burns AF, McGorray S. Glaucoma's impact on quality of life and its relation to clinical indicators. A pilot study. Ophthalmology 1998; 105: 561-566.

4. Gutierrez, P, Wilson MR, Johnson C, Gordon M, Cioffi $G A$, Ritch $R$, et al. Influence of glaucomatous visual field loss on health-related quality of life. Arch Ophthalmol 1997; 115: 777-784.

5. Tripop S, Pratheepawanit N, Asawaphureekorn S, Anutangkoon $W$, Inthayung $S$. Health related quality of life instruments for glaucoma: a comprehensive review. J Med Assoc Thai 2005; 88 Suppl 9: S155-S162.

6. Spaeth G, Walt J, Keener J. Evaluation of quality of life for patients with glaucoma. Am J Ophthalmol 2006; 141 : S3-S14.

7. Alonso J, Espallargues M, Andersen TF, Cassard SD, Dunn E, Bernth-Petersen P, et al. International applicabi- lity of the VF-14. An index of visual function in patients with cataracts. Ophthalmology 1997; 104: 799-807.

8. Nelson-Quigg J, Cello K, Johnson CA. Predicting binocular visual field sensitivity from monocular visual field results. Invest Ophthalmol Vis Sci 2000; 41: 2212-2221.

9. Herdman MJ. Reflexiones sobre la medición de la calidad de vida relacionada con la salud en España. Gac Sanit 2005; 2: 91-92.

10. Siegrist J, Fernández-López JA, Hernández-Mejía $R$. Perspectiva sociológica de la calidad de vida. Med Clin (Barc) 2000; 114: 22-24.

11. Badía X, Carné X. La evaluación de la calidad de vida en el contexto del ensayo clínico. Med Clin (Barc) 1998; 110: 550-556.

12. Cabezas-León M, Gracia-San Roman J, García-Caballero J. Calidad de vida en pacientes intervenidos de catarata. Arch Soc Esp Oftalmol 2005; 80: 449-456.

13. Sánchez Gonzalez R, Yanes Baonza M, Cabrera Majada A, Ferrer Garcia-Borrás JM, Álvarez Nido R, Barrera Linares E. Adaptación transcultural de un cuestionario para medir la calidad de vida de los pacientes con anticoagulación oral. Aten Primaria 2004; 34: 353-359.

14. Sanjuás Benito C. Medición de la calidad de vida: ¿cuestionarios genéricos o específicos? Arch Bronconeumol 2005; 41: 107-109.

15. Llorca J, Martínez-Sanz F, Prieto-Salceda D, FariñasÁlvarez C, Chinchon MV, Quiñones D, et al. Quality of controlled clinical trials on glaucoma and intraocular high pressure. J Glaucoma 2005; 14: 190-195.

16. Knudtson MD, Klein BE, Klein R, Cruickshanks KJ, Lee $K E$. Age-related eye disease, quality of life, and functional activity. Arch Ophthalmol 2005; 123: 807-814.

17. Gupta V, Srinivasan G, Mei SS, Gazzard G, Sihota R, Kapoor KS. Utility values among glaucoma patients: an impact on the quality of life. Br J Ophthalmol 2005; 89: 241-1244.

18. Gupta SK, Viswanath K, Thulasiraj RD, Murthy GV, Lamping DL, Smith SC, et al. The development of the Indian vision function questionnaire: field testing and psychometric evaluation. Br J Ophthalmol 2005; 89: 621-627.

19. Parrish RK 2nd, Gedde SJ, Scott IU, Feuer WJ, Schiffman $J C$, Mangione CM, et al. Visual function and quality of life among patients with glaucoma. Arch Ophthalmol 1997; 115: 1447-1455.

20. Janz NK, Wren PA, Lichter PR, Musch DC, Gillespie BW, Guire KE. Quality of life in newly diagnosed glaucoma patients: The Collaborative Initial Glaucoma Treatment Study. Ophthalmology 2001; 108: 887-897.

21. Nelson P, Aspinall P, O'Brien C. Patients' perception of visual impairment in glaucoma: a pilot study. Br J Ophthalmol 1999; 83: 546-552.

22. Moreno Montañés J, Sainz Gómez C, Moya Molina D, Vásquez López G. Utilidad del test VF-14. Valoración en nuestros pacientes como test preoperatorio de la catarata. Arch Soc Esp Oftalmol 1998; 73: 249-254.

23. Hyman LG, Komaroff E, Heijl A, Bengtsson B, Leske MC. Early Manifest Glaucoma Trial Group. Treatment and vision-related quality of life in the early manifest glaucoma trial. Ophthalmology 2005; 112: 1505-1513.

24. Noe G, Ferraro B, Lamoureux E, Rait J, Keeffe JE. Associations between glaucomatous visual field loss and participation in activities of daily living. Clin Exp Ophthalmol 2003; 31: 482-486. 